\title{
Sensitivity analysis of smart rotor under to uncertainties
}

\author{
Edson H. Koroishi ${ }^{1, *}$, Fabian A. Lara-Molina ${ }^{1, * *}$, and Joana P. Repinaldo ${ }^{1,}$ \\ ${ }^{1}$ Federal University of Technology - Paraná, Cornélio Procópio, PR, CEP 86300-000, Brazil.
}

\begin{abstract}
This contribution encompasses the sensitivity analysis of flexible rotor with active vibration control subjected to uncertainties. The uncertain parameters consist of small variations that are modeled by using random variables and random fields. The structure response subjected to uncertain parameters is analyzed based on the stochastic modeling. Numerical simulations illustrate the structural response of the rotor and their sensitivity indices.
\end{abstract}

\section{Introduction}

Smart rotors are flexible rotors that include a control system to reduce the vibration. These systems are certainly subjected to uncertainties. However, optimal performance is required $[1,2]$. The uncertainties consist of small variations of the mechanical properties, such as damping, stiffness, and geometrical imperfections [3]. Therefore, the examination of parametric uncertainties and the quantification of their effects in the uncertain structural response are necessary in order to predict how the dynamic response varies, by means of numerical methods $[4,5]$. Several methods have been used to analyze effects of the uncertainties in the structural response of flexible rotors: stochastic finite element method [6], fuzzy dynamic analysis [7], fuzzy-stochastic dynamic analysis [1] and polynomial chaos theory [8] among others.

In this contribution, a smart flexible rotor under random uncertainties parameters is analyzed. Additionally, the sensitivity regarding the orbits is examined by using the global-based sensitivity method.

\section{Flexible Rotor Model}

The dynamic response of the considered mechanical system can be represented by using principles of variational mechanics, namely the Hamilton's principle. For this aim, the strain energy of the shaft and the kinetic energies of the shaft and discs are calculated. An extension of Hamilton's principle makes possible to include the effect of energy dissipation. The parameters of the bearings are included in the model by using the principle of the virtual work. For computational purposes, the finite element method is used to discretize the structure so that the energies calculated are concentrated at the nodal points. Shape functions are used to connect the nodal points. In this model, 4 degrees of freedom per node are taken into account, namely two displacements $(u$ and $w)$ and two rotations $(\theta$ and $\psi)$. The Fig. 1 shows the finite element used to model the rotor.

\footnotetext{
*e-mail: edsonh@utfpr.edu.br

**e-mail: fabianmolina@utfpr.edu.br
} 


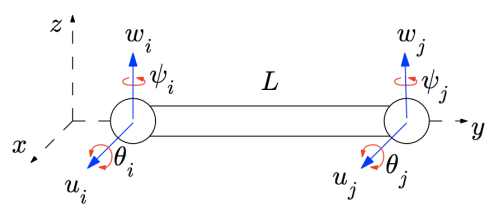

Figure 1. Illustration of the shaft finite element [6].

The following assumptions are considered in the formulation of the model of the flexible rotor: $i$ ) the beam element is modeled according to Euler-Bernoulli's theory, ii) the material presents linear behavior and it is homogeneous and isotropic; and iii) the effects of inertial rotations are taken into account.

According to [3] the model depicted in Fig. 1 can be represented mathematically by the following set of differential equations:

$$
\mathbf{M} \ddot{\boldsymbol{\delta}}(t)+[\mathbf{C}+\phi \mathbf{G}] \dot{\boldsymbol{\delta}}(t)+\mathbf{K} \boldsymbol{\delta}(t)=\mathbf{F}(t)
$$

where $\mathbf{M}=\left(\mathbf{M}_{S}+\mathbf{M}_{D}\right) \in \mathbb{R}^{N \times N}$ and $\mathbf{K}=\left(\mathbf{K}_{S}+\mathbf{K}_{B}\right) \in \mathbb{R}^{N \times N}$ are, respectively, the mass and stiffness matrices, $\mathbf{C}=\left(\mathbf{C}_{B}+\mathbf{C}_{P}\right) \in \mathbb{R}^{N \times N}$ is the damping matrix formed by the contributions of the viscous damping matrix, $\mathbf{C}_{B}$ is the viscous damping matrix of the bearings, and the inherent proportional damping matrix, $\mathbf{C}_{P}=\alpha \mathbf{M}+\beta \mathbf{K}$, and $\mathbf{G}=\left(\mathbf{G}_{D}+\mathbf{G}_{S} \in R^{N \times N}\right.$ designates the gyroscopic matrix formed by the gyroscopic contributions of the rigid discs and the shaft. $\delta(t) \in R^{N}$ and $\mathbf{F}(t) \in R^{N}$ are, respectively, the vectors of the amplitudes of the harmonic generalized displacements and external loads, $\Omega$ is the angular speed of the shaft, and $\alpha$ and $\beta$ represent, respectively, the proportional coefficients of mass and stiffness.

\subsection{Parameterization of the deterministic FE model}

In general, such random variables intervene in a rather complicated manner in the finite element matrices. Hence, for evaluating the variability of the responses associated with these uncertainties, it becomes interesting to perform a parameterization of the FE model, which is understood as a means of making the design parameters factored-out of the elementary matrices. After manipulations, those parameters of interest can be factored-out of the elementary matrices as indicated below:

$$
\mathbf{K}_{S}^{(e)}=E_{S} I_{S} \overline{\mathbf{K}}_{S}^{(e)} \quad \mathbf{K}_{B}^{(e)} \quad=k_{x x} \overline{\mathbf{K}}_{B}^{(e)}+k_{z z} \overline{\mathbf{K}}_{B}^{(e)} \quad \mathbf{C}_{B}^{(e)}=d_{x x} \overline{\mathbf{C}}_{B}^{(e)}+d_{z z} \overline{\mathbf{C}}_{B}^{(e)}
$$

where $\rho_{S}, A_{S}, I_{S}$ and $E_{S}$ represent, respectively, the mass density, the cross-section area, the inertia and the Young's modulus of the shaft. $d_{x x}, d_{z z}$ and $k_{x x}$ and $k_{z z}$ designate, respectively, the damping and stiffness coefficients of the bearings. $\rho_{D}, r_{D}$ and $h_{D}$ represent, respectively, the density, outer radius and thickness of the disks. It is worth mentioning that the matrices appearing in the right hand side of Eq. (2) are those from which the design parameters of interest have been factored-out.

\subsection{Stochastic Modeling}

The stochastic modeling was presented in previous contributions, [6], where additional details can be found. The design parameters which have been factored-out of the matrices appearing 
in Eq. (2) are considered to be random in order to model the system behavior when uncertainties are present in the shaft, disks and bearing elements. The so-called Karhunen-Loève (KL) decomposition, which is a continuous representation for random fields expressed as the superposition of orthogonal random variables weighted by deterministic spatial functions, is used in this paper. A one-dimensional random field $H(y, \theta)$ is defined by its mean value, $E[H(y, \theta)]$, and covariance $C\left(y_{1}, y_{2}\right)=E\left[\left(H\left(y_{1}, \theta\right)-E\left(y_{1}\right)\right)\left(H\left(y_{2}, \theta\right)-E\left(y_{2}\right)\right)\right\}$, where $y$ represents the spatial dependence of the random field, $E[$.] represents the expectation operator, and $\theta$ represents a random process. A single projection on an orthonormal truncated random basis for a one-dimensional homogeneous Gaussian random field is found as it was described previously by [9]:

$$
H(y, \theta)=E(y)+\sum_{r=1}^{n} \sqrt{\lambda_{r}} f_{r}(y) \xi_{r} \theta
$$

where the deterministic functions $f_{r}(y)$ and the scalar values $\lambda_{r}$ are, respectively, the eigenfunctions and the eigenvalues of the covariance $C\left(y_{1}, y_{2}\right)$. Also, the functions $f_{r}(y)$ and the random variables $\xi_{r}(\theta)$ are orthonormal.

The analytical solution of the eigenproblem proposed by [9] for the relatively simple geometry of the one-dimensional rotor model shown in Fig. 1 using the KL expansion into the domain, $\Omega_{y}=\left(y_{1}, y_{2}\right)$, is expressed by:

$$
C\left(y_{1}, y_{2}\right)=\exp \left(\frac{-\left|y_{1}-y 2\right|}{L_{c o r, y}}\right)
$$

where $\left(y_{1}, y_{2}\right) \in[0, L]$ and $L_{c o r, y}$ indicates the correlation length indicates the correlation length characterizing the decreasing behavior of the covariance with the distance between the observation points in the direction. Also, it can be noted that this continuous model of covariance function corresponds to a homogeneous (stationary) random field. The expansion detailed previously has been chosen in order to model the elementary random stiffness matrices of the shaft element, as follows:

$$
\mathbf{K}_{S}^{(e)}(\theta)=\mathbf{K}_{S}^{(e)}+\sum_{r=1}^{n} \overline{\mathbf{K}}_{S r}^{(e)} \xi_{r}(\theta)
$$

\subsection{Sensitivity Analysis}

The sensitivity analysis aims at determining the influence of each uncertain parameter of the flexible shaft and the bearings on the dynamic response of the rotor system. Consequently, this analysis allows indicating the degree of influence of each parameter on the variation of the structural response of the rotor.

Among the various methods used to analyze the sensitivity, the variance-based sensitivity analysis decomposes the variance of the output of the model into fractions which are associated with the variation of each parameter [10]. This method allows quantifying the effect of the variety of every individual parameter on the dynamic response of the robot by using a probabilistic framework based on the Monte Carlo Simulation method. Additionally, this method copes with nonlinear models, which is suitable to quantify the sensitivity of the smart rotor.

Considering the model under the form $y=f(\mathbf{w})$, where $y$ is a scalar output and $\mathbf{w}=$ $\left[\begin{array}{lll}w_{1} & \cdots & w_{k}\end{array}\right]^{T} \in \mathbb{R}^{k \times 1}$ is a vector of $k$ uncertain parameters, for this contribution $w=$ $\left[\begin{array}{lllllllll}E_{s} & k_{x 1} & k_{z 1} & k_{x 2} & k_{z 2} & c_{x 1} & c_{z 1} & c_{x 2} & c_{z 2}\end{array}\right]$. These parameters are considered as independently and uniformly distributed within the unit hypercube, i.e., $w_{i} \in[0,1]$ for $i=1, \ldots, k$. 
$f(\mathbf{w})$ is decomposed:

$$
y=f(\mathbf{w})=f_{0}+\sum_{i=1}^{k} f_{i}\left(w_{i}\right)+\sum_{i<j}^{k} f_{i j}\left(w_{i}, w_{j}\right)+\cdots+f_{12 \ldots, k}
$$

The decomposition of the variance expression is [11]:

$$
V(y)=\sum_{i=1}^{k} V_{i}+\sum_{i<j}^{k} V_{i j}+\cdots+V_{12 \ldots k}
$$

where $V_{i}=V_{w_{i}}\left(E_{\mathbf{w}_{\sim i}}\left(y \mid w_{i}\right)\right), V_{i j}=V_{w_{i j}}\left(E_{\mathbf{w}_{\sim i j}}\left(y \mid w_{i j}\right)\right)$, and so on. A variance based first order effect for a generic design parameter $w_{i}$ is $V_{w_{i}}\left(E_{\mathbf{w}_{\sim i}}\left(y \mid w_{i}\right)\right)$, where $w_{i}$ is the i-th parameter and $\mathbf{w}_{\sim i}$ denotes the matrix of all parameters except $w_{i}$. The meaning of the inner expectation operation is that the mean of $y$ is taken over all possible values $\mathbf{w}_{\sim i}$ while keeping $w_{i}$ fixed. The associated sensitivity measure denominated first-order sensitivity index is defined as:

$$
s_{i}=\frac{V_{w_{i}}\left(E_{\mathbf{w}_{\sim} i}\left(y \mid w_{i}\right)\right)}{V(y)}
$$

$s_{i}$ states the effect of the variation of $w_{i}$ only, however divided by the variation in other parameters. Nevertheless, the total effect-index $s_{T i}$ measures the contribution to the output variance of $w_{i}$, including all the effects of its interactions with any other input parameter.

$$
s_{T i}=\frac{E_{\mathbf{w}_{\sim i}}\left(V_{w_{i}}\left(y \mid w_{\sim i}\right)\right)}{V(y)}=1-\frac{V_{\mathbf{w}_{\sim i}}\left(E_{w_{i}}\left(y \mid w_{\sim i}\right)\right)}{V(y)}
$$

The Monte Carlo Simulation combined with the Latin Hypercube sampling [12] is used to calculate the total-effect indices. The total number of model evaluation to compute the totalsensitivity index is $N=n_{s}(k+1)$, where $n_{s}$ is the number of the Monte Carlo samples [10].

\section{Numerical Results}

The rotor's model is represented by the set of differential equations as stated by [3] in Eq. (1), this model was obtained by discretizing the shaft into 32 Euler-Bernoulli beam elements. The disks $D_{1}$ and $D_{2}$ are located at nodes \#13 and \#22, the bearings $B_{1}$ (Hybrid Bearing) and $B_{2}$ are located at nodes \#4 and \#31 and the sensors to measure the two orthogonal displacement (along $x$ and $z$ axis) located at nodes \#8 and \#27.

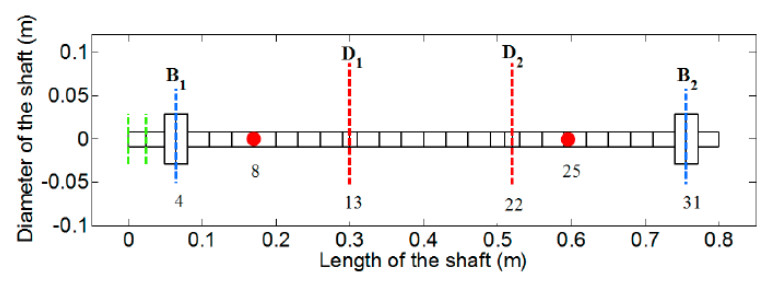

Figure 2. Finite Element model of the rotor.

The parameters of the bearings were determined experimentally in previous works [13].In order to evaluate the effect of uncertain parameters in the flexible rotor the orbits of the shaft 
were computed considering $\% 5$ of dispersion in the uncertain parameters: Youngs modulus of the shaft, and the stiffness and damping of the bearings. Consequently, eleven uncertain parameters are considered in this contribution, thus $E_{S}, k_{x 1}, k_{z 1}, k_{x 2}, k_{z 2}, c_{x 1}, c_{z 1}, c_{x 2}, c_{z 2}$. The computations of the stochastic stiffness matrix of the shaft element is performed by assuming the correlation length $L_{c o r, y}=0.02725 \mathrm{~m}$, corresponding to the length of the beam elements, according to FE mesh.

\subsection{Uncertain Structural Response}

The time domain analysis of the rotor with uncertain parameters was computed by considering a rotation speed of 1600 RPM in which the rotor operates above its first two critical speeds. Figure 3 shows that the orbits by using the LQR controller have a larger variation than the orbits obtained with (Linear Quadratic Regulator) LQR using (Linear Matrix Inequalities) LMIS .

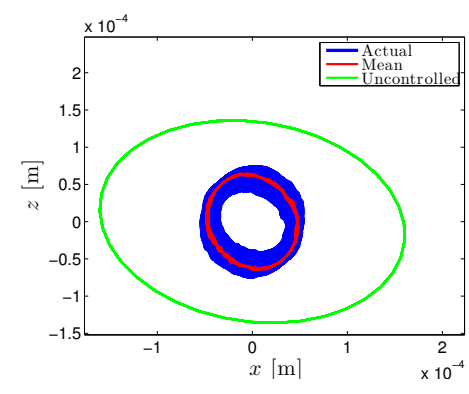

(a)

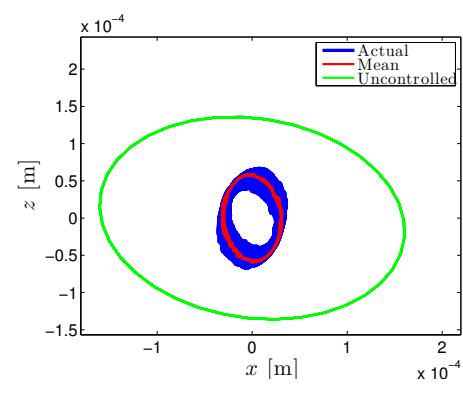

(b)

Figure 3. Orbits: (a) LQR, (b) LQR using LMIS.

\subsection{Sensitivity Analysis}

The sensitivity analysis is applied in order to determine the contribution of every single uncertain parameter in the uncertain orbits presented in Fig. 3. The maximum radius of the orbits as considered as the output of the model to evaluate the sensitivity, thus: $\max _{t} r(t)=\sqrt{x(t)^{2}+z(t)^{2}}$, where $x(t)$ and $z(t)$ are the coordinated of the orbits. Addition$t$
ally, the set of uncertain parameters considered in this analysis is the same as was used in the uncertain analysis.

The orbits of the rotor are evaluated at a constant rotational velocity. Moreover, the gravity is acting in the $z$. Thus, the sensitivity indexes show that the most sensitive parameter in the uncertain response of the orbits is the Young modulus of the shaft $E_{s}$ (see Fig. 4). Additionally the stiffness in the $z$ axis direction of first bearing $B_{1}$ also exhibits a significant sensitivity in the uncertain orbits response. The EMA damps the controlled system, thus the uncertain damping of the the bearings is not sensitive in the uncertain orbits. Additionally, the uncertain masses of the disks $m_{D 1}$ and $m_{D 2}$ are not sensitive because the steady response is evaluated, i.e., with constant velocity and acceleration. As presented previously, the controllers influence the dispersion of the orbits. However, the sensitivity of the orbits is not affected. 


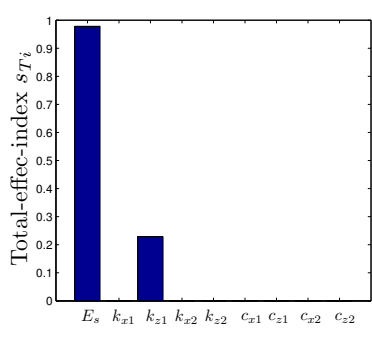

(a)

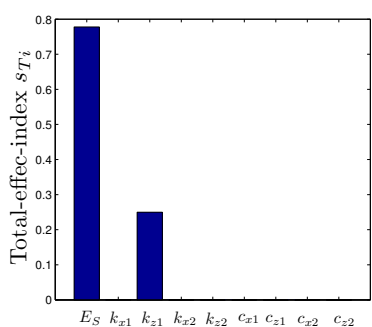

(b)

Figure 4. Sensitivity effects: (a) LQR, (b) LQR using LMIS.

\section{Conclusions}

This contribution illustrated a numerical method that permits to evaluate the effects of uncertainties on the structural response of a smart rotor system and also to assess the sensitivity of every uncertain parameter. The numerical results permit to identify how every uncertain parameter contributes to the uncertain response of orbits. One can observe that the stiffness of the shaft is the most sensitive parameter followed by the stiffness of the bearings.

The authors gratefully acknowledge the financial support for this research from CNPq (Process 402581/2016-4), Araucária Foundation and CAPES.

\section{References}

[1] F.A. Lara-Molina, E.H. Koroishi, V. Steffen Jr, Latin American Journal of Solids and Structures 12, 1807 (2015)

[2] C. Fu, X. Ren, Y. Yang, W. Qin, Nonlinear Dynamics 89, 2115 (2017)

[3] M. Lalanne, G. Ferraris, Rotordynamics Prediction in Engineering (John Wiley \& Sons, 1998)

[4] F. Lara-Molina, E. Koroishi, D. Dumur, V. Steffen, IFAC-PapersOnLine 48, 214 (2015), 11th IFAC Symposium on Robot Control SYROCO 2015

[5] E.S. de Cursi, R. Sampaio, Uncertainty quantification and stochastic modeling with matlab (Elsevier, 2015)

[6] E.H. Koroishi, A.A. Cavalini Jr., A.A.M.G.d. Lima, V. Steffen Jr., Journal of the Brazilian Society of Mechanical Sciences and Engineering 34, 574 (2012)

[7] A.A. Cavalini Jr, F.A. Lara-Molina, T.d.P. Sales, E.H. Koroishi, V. Steffen Jr, Latin American Journal of Solids and Structures 12, 1487 (2015)

[8] J. Didier, B. Faverjon, J.J. Sinou, Journal of Vibration and Control 18, 712 (2012)

[9] R.G. Ghanem, P.D. Spanos, Stochastic finite elements: a spectral approach (Courier Corporation, 2003)

[10] A. Saltelli, M. Ratto, T. Andres, F. Campolongo, J. Cariboni, D. Gatelli, M. Saisana, S. Tarantola, Global Sensitivity Analysis: The Primer (John Wiley \& Sons Chichester, England, 2008)

[11] I.M. Sobol', Matematicheskoe Modelirovanie 2, 112 (1990)

[12] A. Florian, Probabilistic engineering mechanics 7, 123 (1992)

[13] E. Koroishi, F. Lara-Molina, A. Borges, V. Steffen, Journal of Vibration and Control pp. $1-12(2015)$ 